tery pulsation, are not unexpected and do not require comment. Surprisingly no apparent trend was found with overweight and high blood pressure; perhaps both these features were so common that they ceased to have any discriminatory value.

The comparison between the newly discovered diabetics and the established diabetics (over 10 years) is probably of limited value in that current prevalence only is being compared and high-risk persons will have been removed from both groups by previous fatal coronary heart disease. Within the surviving established diabetics (over 10 years' duration of disease), however, a higher prevalence of coronary heart disease was found in those with milder, adult-onset type diabetes controlled by diet or oral drugs as compared with the insulin-dependent diabetics. That finding when combined with the high prevalence of ischaemic heart disease at initial diagnosis in late-onset diabetics suggests that this type of patient may account for much ischaemic heart disease in diabetics. Such a finding may not have been discernible before the use of oral drugs, since many of these patients were treated with insulin and the essentially mild nature of their carbohydrate intolerance would have escaped detection.

\section{REFERENCES}

Blackburn, H., Keys, A., Simonson, E., Rautaharju, P., and Punsar, S. (1960). Circulation, 21, 1160.
Blotner, H. (1930). New England Fournal of Medicine, 203, 709.

Bradley, R. F., and Bryfogle, J. W. (1956). American foumal of Medicine, $20,207$.

Bridges, J. M., Dalby, A. M., Millar, J. H. D., and Weaver, J. A. (1965). Lancet, 1, 75 .

Clawson, B. J., and Bell, E. T. (1949). Archives of Pathology, 48, 105.

Epstein, F. H. et al. (1965). Annals of Internal Medicine, 62, 1170.

Hayward, R. E., and Lucena, B. C. (1965). Fournal of the lnstitute of Actuaries, 91, 286.

Keen, H., et al. (1965). Lancet, 2, 505.

Liebow, I. M., Hellerstein, H. K., and Miller, M. (1955). American

Liebow, I. M., Newill, V. A., and Oseasohn, R. (1964). American fournal of Medical Sciences, 248, 403.

Lisa, J. R., Magiday, M., Galloway, I., and Hart, J. F. (1942). fournal of the American Medical Association, 120, 192.

Mayne, E., Bridges, J. B., and Weaver, J. A. (1970). Diabetologia. In press.

Millard, E. B., and Root, H. F. (1948). American fournal of Digestive Diseases, 15, 41.

Mitchell, J. R. A., and Schwartz, C. (1965). Arterial Disease, p. 79. Oxford, Blackwell.

Nathanson, M. H. (1932). American fournal of Medical Sciences, 183, 495.

Oakley, W. G., Pyke, D. A., and Taylor, K. W. (1968). Clinical Diabetes, p. 512 . Oxford, Blackwell.

Root, H. F., Bland, E. F., Gordon, W. H., and White, P. D. (1939). fournal of the American Medical Association, 113, 27.

Root, H. F., and Sharkey, T. P. (1936). New England foumal of Medicine, 215,605 .

Shepardson, H. C. (1930). Archives of Internal Medicine, 45, 674.

Stearns, S., Schlesinger, M. J., and Rudy, A. (1947). Archives of Internal Medicine, 80, 463.

Warren, S., and LeCompte, P. M. (1952). The Pathology of Diabetes Mellitus, 3rd ed., p. 138. Philadelphia, Lea and Febiger.

Zlatkis, A., Zak, B., and Boyle, A. J. (1953). Fournal of Laboratory and Clinical Medicine, 41, 486.

\title{
Study of Membrane Permeability in Haemodialysis
}

\author{
D. B. EVANS, ${ }^{*}$ M.R.C.P. ; J. G. LINES, † PH.D.
}

\begin{abstract}
Cummary: The efficiency of haemodialysis has been determined when a Watson-Marlow (Kiil) dialyser was used repetitively without changing the membranes. Dialysis efficiency was assessed from the clearance of creatinine and of urea from the patient's blood. No significant deterioration in dialysis could be detected in six dialyses through the same membrane. Leaving the dialyser unit for up to eight hours before washing it out after the completion of each dialysis did not appear to have any effect on dialysis efficiency.

It was concluded that repetitive haemodialysis through the same membranes is possible, but careful monitoring of the patient to assess suitability for such treatment, and subsequently at each dialysis, is essential.
\end{abstract}

\section{Introduction}

The dialyser module of present-day artificial kidneys, the Kiil dialyser, consists of three polypropylene boards with grooved surfaces, between each pair of which are placed a pair of membranes. The patient's blood circulates between the membrane sheets, and dialysate fluid, prepared either in tanks or by a proportioning machine, flows over the outer surface of the membranes counter-current to the blood.

Electrolytes and low molecular weight constituents of the blood plasma pass through the pores of the membrane into the dialysis fluid down a concentration gradient. The rate of clearance of these substances from the blood is known as the

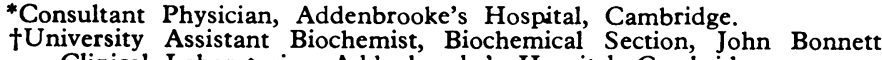
Clinical Laboratories, Addenbrooke's Hospital, Cambridge. dialysance (D) of that molecule or ion and can be calculated from the equation (Wolf $e t$ al.):

$$
D=\frac{a(A-V)}{A}
$$

where $\mathrm{a}=$ blood flow rate, $\mathrm{A}=$ arterial blood level, and $\mathrm{V}=$ venous blood level.

For patients maintained on haemodialysis a 14-hour period of dialysis usually results in a decrease of plasma urea from about 150 to about $40 \mathrm{mg} . / 100 \mathrm{ml}$. and of creatinine from about 10 to $3 \mathrm{mg}$. $/ 100 \mathrm{ml}$.

With the increase in size of hospital dialysis units and the development of home dialysis, more economical use of resources, in both material and time, has become essential. Haemodialysis has conventionally involved use of new membranes in the dialyser unit for each dialysis. Shaldon and Oakley (1967) practised washing the dialyser units through when dialysis was complete and then reusing the dialyser up to six times. The dialyser unit would thus require rebuilding much less frequently, probably only once every two to three weeks.

This study was undertaken to determine whether there was any decrease in the efficiency of dialysis with reusage of membranes. The effects of leaving the dialyser for up to eight hours after completing dialysis before washing it out were also determined, because it is not always convenient or possible to begin the washing procedure immediately the haemodialysis is completed.

\section{Methods and Materials}

Twice-weekly haemodialysis was performed with a modified Kiil dialyser (Watson-Marlow) and PT 150 cuprophane membranes, a warm $37^{\circ} \mathrm{C}$. single-pass system being used. The dialysis was usually of 14 hours' duration. Arterial 
and venous blood specimens were collected 10 minutes after the beginning of dialysis and at $1,4,7$, and 10 hours. The blood flow rate through the dialyser was measured at each of these times by an air-bubble method (Scribner). Each specimen of blood plasma was analysed for urea and creatinine by standard autoanalyser methodology.

Within half an hour of completing the dialysis the dialyser was disconnected from the dialysate source and the blood and dialysate were allowed to drain. The blood and dialysis compartments were then connected by a hose to a mains water source and flushed through for half an hour. The whole dialyser unit was then tested for air leaks to $200 \mathrm{~mm} . \mathrm{Hg}$ and, if intact, filled with $5 \%$ formalin and allowed to stand until required for the next dialysis. It must be emphasized that for these purposes the one dialyser was always reserved for the same patient during the whole of the test. In all, six dialyses were performed through the same dialyser unit before dismantling it and replacing the membranes. In subsequent investigations, periods of either four or eight hours were allowed to elapse before washing out the dialyser after the completion of haemodialysis.

\section{Results}

Repetitive use of the same membrane did not appear to have a significant effect on the rates of clearance of either urea or creatinine, as is shown in the Table. These clearances

Rates of Clearance

\begin{tabular}{|c|c|c|c|c|c|c|c|c|c|c|c|c|c|c|}
\hline \multirow{3}{*}{\multicolumn{2}{|c|}{$\begin{array}{c}\text { Time of } \\
\text { Sampling }\end{array}$}} & & \multicolumn{12}{|c|}{ Clearance ( $\mathrm{ml} . / \mathrm{min})}$. \\
\hline & & & \multicolumn{6}{|c|}{ Urea Dialysis } & \multicolumn{6}{|c|}{ Creatinine Dialysis } \\
\hline & & & A & B & C & D & $\mathrm{E}$ & $\mathrm{F}$ & $\mathbf{A}$ & B & $\mathrm{C}$ & D & $\mathbf{E}$ & F \\
\hline $\begin{array}{l}10 \text { min. } \\
1 \text { hour } \\
4 \text { hours } \\
7 \text { hours } \\
10 \text { hours }\end{array}$ & $\begin{array}{l}. . \\
\ldots \\
\ldots \\
\cdots\end{array}$ & $\begin{array}{l}\cdots \\
\cdots\end{array}$ & $\begin{array}{l}99 \\
97 \\
67 \\
72 \\
66\end{array}$ & $\begin{array}{l}76 \\
63 \\
68 \\
59\end{array}$ & $\begin{array}{l}85 \\
99 \\
72 \\
68 \\
54\end{array}$ & $\begin{array}{l}61 \\
64 \\
59 \\
57 \\
52\end{array}$ & $\begin{array}{l}68 \\
68 \\
68 \\
74 \\
62\end{array}$ & $\begin{array}{l}66 \\
66 \\
69 \\
62 \\
66\end{array}$ & $\begin{array}{l}73 \\
66 \\
47 \\
56 \\
48\end{array}$ & $\begin{array}{l}62 \\
76 \\
53 \\
50\end{array}$ & $\begin{array}{l}67 \\
78 \\
59 \\
55 \\
58\end{array}$ & $\begin{array}{l}51 \\
54 \\
45 \\
47 \\
47\end{array}$ & $\begin{array}{l}57 \\
59 \\
55 \\
50 \\
43\end{array}$ & $\begin{array}{l}52 \\
51 \\
50 \\
44 \\
48\end{array}$ \\
\hline
\end{tabular}

A, B, C, etc., represent consecutive dialyses using the same membrane.

represent decreases in metabolite concentration of from about 150 to $50 \mathrm{mg}$. $/ 100 \mathrm{ml}$. for urea and from 10 to $4 \mathrm{mg} . / 100 \mathrm{ml}$. for creatinine (Fig. 1). The differences in initial values of each dialysis reflect whether a three- or four-day period separated consecutive dialyses.

The effects of standing for four or eight hours before washing out the dialyser are shown in Fig. 2. To eliminate the

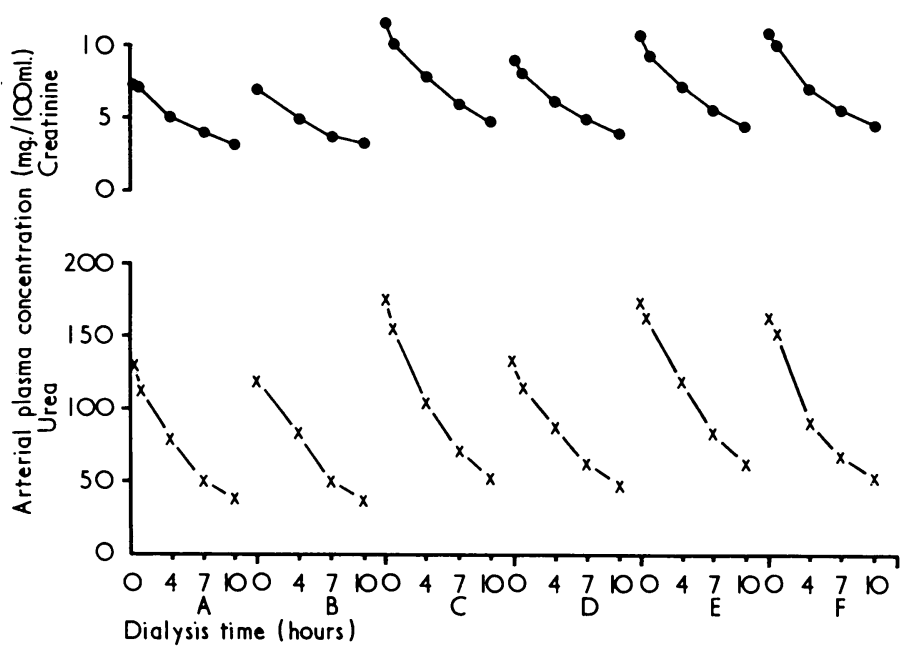

Fig. 1.-Each curve represents the fall in arterial plasma urea or creatinine during the course of haemodialysis-blood samples having been taken at 10 minutes, $1,4,7$, and 10 hours after commencement of dialysis. A, B, C, D, E, F, represent consecutive dialyses with same membrane.
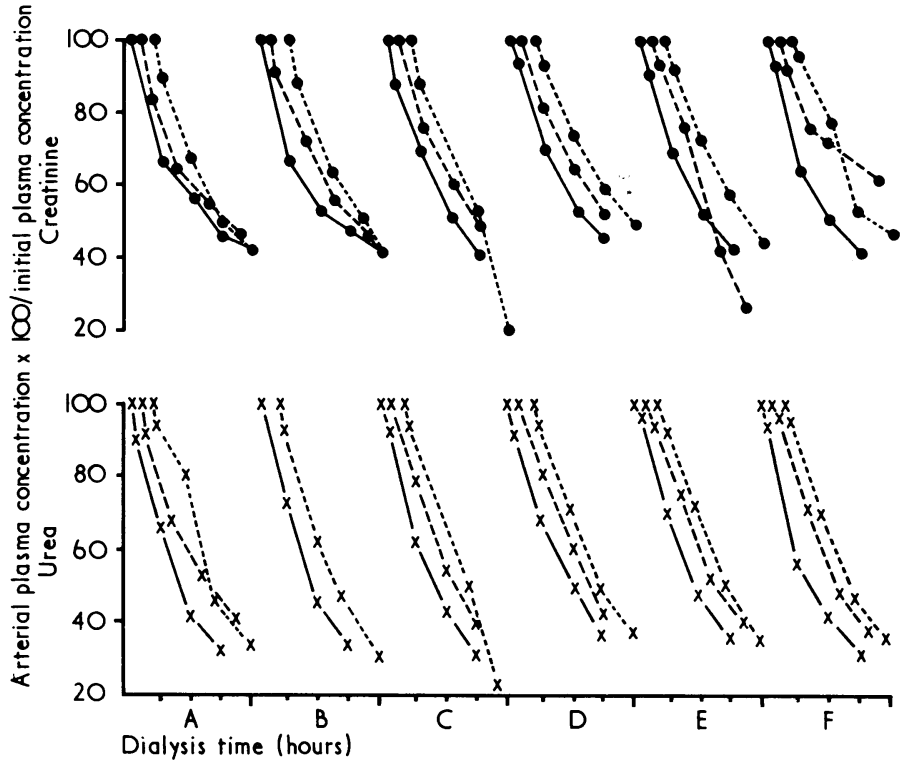

Fig. 2.-Percentage reduction in plasma arterial concentration for urea and creatinine during consecutive dialysese. $0-0$ membrane washed and creatinine during consecutive dialysese. o membrane washed four immediately after end of dialysis. o- - o membrane washed four
hours after end of dialysis. o..... membrane washed eight hours after end of dialysis.

effect of variations in initial arterial concentration of metabolites the concentration of metabolite in the specimens at 1,4 , 7 , and 10 hours has been expressed as a percentage of the initial concentration.

\section{Discussion}

The results show that it is possible to reuse a dialyser up to at least five times without any significant decrease in dialysis efficiency, and it is also apparent that a time lag between completing the dialysis and washing out the dialyser does not affect the efficiency.

Thrombocytes, leucocytes, fibrin, and other proteinaceous substances may adhere to the blood surface of the membrane. Such adhesions would reduce the effective surface area of the membrane and decrease dialysis efficiency. Clearly there was no such effect in the dialysis reported here, other than a possible initial slight clogging of the membrane on first usage (see Table), as clearance rates for urea reached the high values attained in the first hour ( 99 and $97 \mathrm{ml}$./minute) on only one subsequent occasion. As the extent of the pore clogging is most probably dependent on the blood composition of the patient being dialysed, strict monitoring of dialysis efficiency must be performed when repetitive dialyses through the same membranes are undertaken. For this purpose a pre-dialysis and post-dialysis analysis of plasma urea or creatinine would suffice. The type of kidney used for repetitive dialyses through the same membrane also appears to be important; a low residual blood volume (with the Watson-Marlow kidney it is usually less than $5 \mathrm{ml}$.) (Evans et al., 1967) and the ability to wash out all macroscopic constituents are essential. Finally, the greatest care is essential in aseptic storage and testing the membranes for leaks before reuse, and reuse of a dialyser unit must be by the same patient.

We wish to thank the nursing staff and technicians of the renal unit for collection of specimens, the department of medical photography for the charts, and Mrs. J. Small for preparing the typescript. fournal, 4, 651 . tation. Proceediney, J. J. (1967). In Dialysis and Renal Transplantation: Proceedings of the 4th Conference of the European Dialysis and Renal Transplant Association, edited by D. N. S. Kerr, p. 24. Amsterdam, Excerpta Medica.

Wolf, A. V., Remp, D. G., Kiley, J. E., and Currie, G. D. (1951). 